\title{
Phase Transition Driven Discontinuity in Thermodynamic Size Selection
}

\author{
R. van Gastel, ${ }^{1}$ D. Kaminski, ${ }^{2,3}$ E. Vlieg, ${ }^{2}$ and B. Poelsema ${ }^{1}$ \\ ${ }^{1}$ Physics of Interfaces and Nanomaterials, MESA Institute for Nanotechnology, University of Twente, P.O. Box 217, \\ 7500 AE Enschede, The Netherlands \\ ${ }^{2}$ Institute for Molecules and Materials, Radboud University Nijmegen, Heyendaalseweg 135, 6525 AJ Nijmegen, The Netherlands \\ ${ }^{3}$ Department of Chemistry, University of Life Sciences in Lublin, Akademicka 13, 20-950 Lublin, Poland
}

(Received 19 June 2012; published 7 November 2012)

\begin{abstract}
We show how an order-disorder phase transition in a two-dimensional system can discontinuously alter the shape and size of stress-stabilized self-assembled nanostructures. Low energy electron microscopy was used to study the dealloying of the $\mathrm{Cu}(111)-\sqrt{3} \times \sqrt{3}-R 30^{\circ}-\mathrm{Bi}$ surface alloy. The gradual expulsion of embedded bismuth from the alloy with increasing temperature induces a hard-hexagon-type orderdisorder transition in the surface alloy. Our low energy electron microscopy results demonstrate how the loss of long-range order induces enormous changes in the domain patterns that the alloy forms with a $\mathrm{Bi}$ overlayer phase. We propose that the occurrence of phase transitions in one of the two surface phases that constitute a self-assembled domain pattern, provides a general, largely unexplored, mechanism that can be used to influence the morphological details of two-dimensional nanostructures.
\end{abstract}

DOI: 10.1103/PhysRevLett.109.195501

Surface stress is one of the physical phenomena that is being actively investigated to aid in the stabilization of nanostructures [1-10]. Its application to achieve thermodynamic nanoscale size selection offers the prospect of manipulating the equilibrium size of nanostructures in a controlled manner [11-17]. A mismatch in stress between two surface phases will lead to the formation of bulk strain fields at the boundaries between the phases $[18,19]$. The elastic energy associated with the strain fields has been demonstrated to act as a stabilizing force for several different types of two-dimensional periodic domain structures [20-25], as well as isolated surface domains [13,14]. Temperature and coverage can be used as experimental variables to carefully manipulate the balance between the elastic energy and the domain boundary free energy to achieve size and shape selection.

Whereas a phase transition itself can already lead to the formation of a self-assembled domain pattern [13,26,27], a structural phase transition in one of the two surface phases that constitute a domain pattern can discontinuously alter the energetics and appearance of a pattern. Here we describe a surprising twist to the behavior of the $\mathrm{Bi} / \mathrm{Cu}(111)$ system that involves a second-order phase transition. $\mathrm{Bi} / \mathrm{Cu}(111)$ is quite similar to the previously studied $\mathrm{Pb} / \mathrm{Cu}(111)$ system $[6,28]$. When $\mathrm{Bi}$ is deposited on $\mathrm{Cu}$ (111) it initially forms a random surface alloy [29]. Upon reaching a Bi coverage of $1 / 3 \mathrm{ML}$ (expressed in $\mathrm{Cu}$ unit cells), the surface alloy assumes an ordered $\sqrt{3} \times \sqrt{3}-R 30^{\circ}$ structure and any additional $\mathrm{Bi}$ that is deposited in excess of $1 / 3 \mathrm{ML}$ is incorporated in an overlayer phase [30,31]. The precise structure of the overlayer phase depends on coverage and temperature and has been described elsewhere [32,33]. In what follows we will refer to the $\sqrt{3} \times$ $\sqrt{3}-R 30^{\circ}-\mathrm{Bi}$ surface alloy as the $\sqrt{3}$ alloy. The overlayer
PACS numbers: 81.16.Dn, 68.35.Md, 68.37.Nq, 68.47.De

and $\sqrt{3}$ alloy self-assemble into periodic domain structures.

Low energy electron microscopy (LEEM) [34] was used to monitor the temperature dependent evolution of the selfassembled domain patterns of the two $\mathrm{Bi}$ phases and characterize the structure of the two domain types in both real and reciprocal space. For our experiments a $\mathrm{Cu}$ single crystal was mechanically polished and aligned to the (111) crystallographic direction with an accuracy better than $0.1^{\circ}$. The $\mathrm{Cu}(111)$ surface was further prepared by repeated cycles of $\mathrm{Ar}^{+}$-ion bombardment and annealing until LEEM images revealed large defect-free terraces. Bi was vapor deposited from a Knudsen cell.

The expected temperature dependence of the domain size $l_{0}$ is given by the following relation:

$$
l_{0}=\pi a e^{\left[2 \pi E \beta /(\Delta \sigma)^{2}(1-\nu)\right]+1}
$$

where $\beta$ is the domain boundary free energy, $\Delta \sigma$ is the stress mismatch between the two phases, $E$ is Young's modulus, $\nu$ is the Poisson ratio and $a$ is the domain boundary width $[1,2]$. With increasing temperature the entropic contribution to the domain boundary free energy will dominate, which eventually leads to a vanishing domain size. A sequence of LEEM images illustrating the temperature dependence of the evolution of the equilibrated domain pattern of the overlayer and $\sqrt{3}$ alloy phase of $\mathrm{Bi} / \mathrm{Cu}(111)$ is shown in Fig. 1. Bi was initially deposited at a substrate temperature below $573 \mathrm{~K}$ up to a coverage of $0.376 \pm 0.001 \mathrm{ML}$, where the $\sqrt{3}$ alloy coexists with the overlayer phase. At that temperature the overlayer phase is in a liquid state and no longer shows any long-range ordering [33]. Figures 1(a)-1(f) illustrate the behavior of the $\sqrt{3}$ alloy (dark) and the overlayer (bright) as the temperature of the substrate is slowly ramped through $670 \mathrm{~K}$. Initially, in 

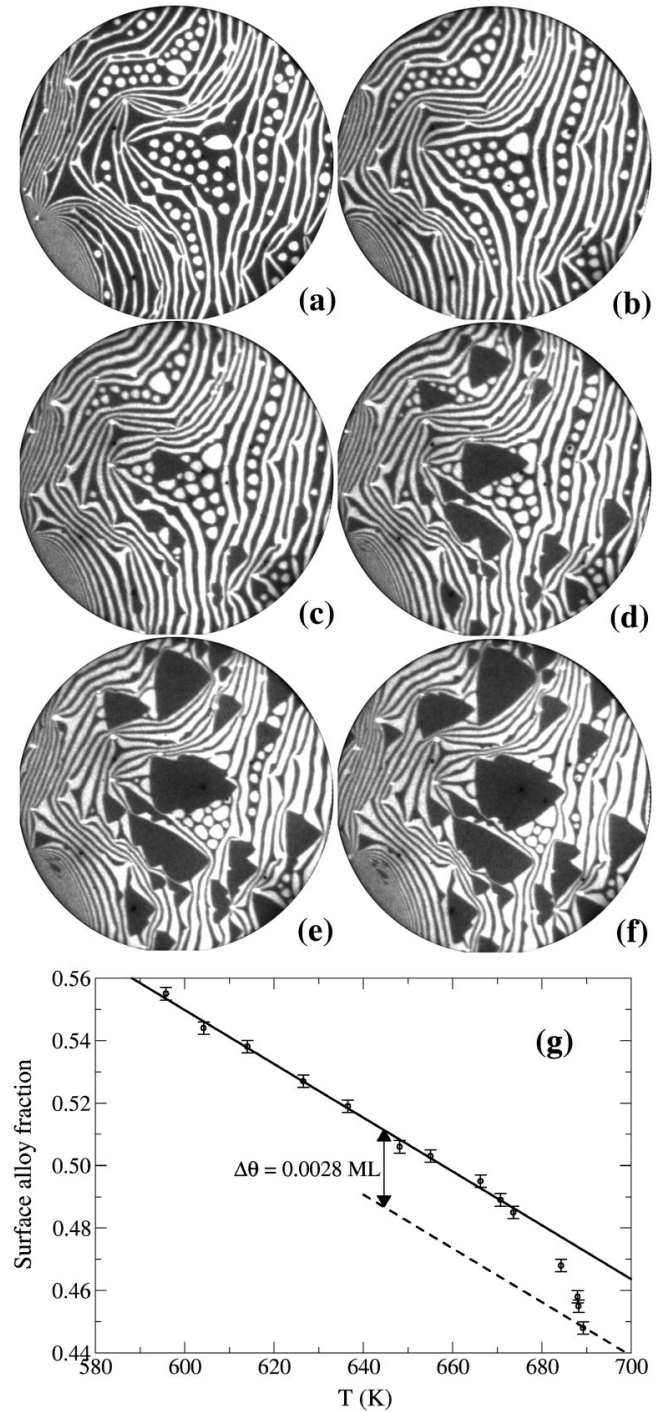

FIG. 1. $4 \mu \mathrm{m}$ field of view (FoV), 18.1 V LEEM images of the dealloying $\sqrt{3}$ alloy at a coverage of $0.376 \mathrm{ML}$, where it coexists with a liquid overlayer phase. [(a), $t=0 \mathrm{~s}, \mathrm{~T}=604 \mathrm{~K}]$ Starting situation where the dark $\sqrt{3}$ alloy domains coexists with the bright overlayer phase. At this coverage the overlayer phase consists of monolayer high islands in a matrix of the $\sqrt{3}$ alloy phase. In the center of the image a terrace is populated by many overlayer islands. Near the edges of the image a zebralike pattern is observed where the $\mathrm{Bi}$ overlayer decorates steps of the $\mathrm{Cu}$ substrate. Cusps in these stripes mark translational domain boundaries of the $\sqrt{3}$ phase. $[(\mathrm{b}), \mathrm{t}=694 \mathrm{~s}, \mathrm{~T}=671 \mathrm{~K}]$ At constant coverage, but increased temperature the shape of the overlayer domains becomes slightly more triangular. The relative area fraction occupied by the overlayer phase has also slightly increased. [(c), $t=835 \mathrm{~s}, \mathrm{~T}=684 \mathrm{~K}]$ The first signs of a discontinuous change in the domain pattern become visible as large triangular domains appear in the $\sqrt{3}$ phase. [(d)-(f), $\mathrm{t}=875-1029 \mathrm{~s}, \mathrm{~T}=685 \mathrm{~K}]$ The alloy domains grow far beyond the original feature size and assume a distinctly triangular shape. (g) The relative area fraction of the $\sqrt{3}$ alloy phase as a function of temperature. The transition of the $\sqrt{3}$ phase is complete at a temperature of $690 \mathrm{~K}$. The deviation from the initial linear decrease corresponds to a coverage discontinuity of $0.0028 \mathrm{ML}$, indicated by the dashed line. panels (a) and (b), a small, but measurable increase in size of the domains is observed, accompanied by a slight decrease in the relative coverage of the $\sqrt{3}$ phase, quantified in Fig. 1(g). The changing relative areas indicate a change in Bi coverage of either of the two phases. Simultaneously, a slight deformation of the domains into a more triangular shape becomes visible. Above $674 \mathrm{~K}$, distinctly triangular domain shapes develop that eventually dominate the appearance of the domain pattern in Fig. 1(f).

The observations of Figs. 1(c)-1(f) clearly violate the expectation of a simple temperature dependent domain size [6] and shape [13]. Instead, a different effect dominates the energetics of the domain pattern. We propose that this effect is a hard-hexagon type order-disorder transition [35-37] that occurs in the $\sqrt{3}$ alloy as a result of temperature dependent de-alloying. The occurrence of this transition is hinted at by the data shown in Fig. 1(g). Prior to reaching $674 \mathrm{~K}$, we observe that the relative area fractions of the two surface phases change slightly with temperature. At $604 \mathrm{~K}$, the alloy and the overlayer phase occupy a fraction of the visible surface area of 0.541 and 0.458 , respectively. At $671 \mathrm{~K}$ this has changed to a relative occupation of 0.495 and 0.505 , respectively, without a change in the total Bi coverage. An accelerated variation of the area fractions corresponding to a discontinuity in Bi coverage of the $\sqrt{3}$ phase of $0.0028 \mathrm{ML}$ is visible in the right hand side of the graph where the abrupt changes in the pattern occur. We note that the loss of $\mathrm{Bi}$ through desorption from the surface does not become significant on the time scale of our experiments until a temperature of $800 \mathrm{~K}$. To interpret the change in relative area fraction we assume that it consists of two contributions: a small linear expansion of the overlayer phase and a decreasing Bi occupation of the $\sqrt{3}$ lattice in the $\sqrt{3}$ alloy. Since the observations of Fig. 1 do not allow us to separate out the two contributions, we need to accurately characterize the structure of the $\sqrt{3}$ phase both below and above $680 \mathrm{~K}$.

To elucidate how the structure of the $\sqrt{3}$ alloy changes with temperature we used. Figure 2(a) shows a LEEM image that was recorded after the deposition of $0.399 \mathrm{ML}$ of $\mathrm{Bi}$ at $537 \mathrm{~K}$. The sample was heated to a temperature of $755 \mathrm{~K}$ to induce the phase transition and subsequently quenched. Some of the Bi became incorporated in stable three-dimensional structures, lowering the effective $\mathrm{Bi}$ coverage. After quenching, the realloying of $\mathrm{Bi}$ becomes kinetically limited and for a short period of time the two phases coexist. The $\mu$ LEED patterns that were obtained on both phases are shown in Figs. 2(b) and 2(c). Both yield the same $\sqrt{3} \times \sqrt{3}$ structure; however, the LEEM contrast of Fig. 2(a) implies that the $I / V$ characteristic, and consequently the Bi coverage of both phases, is distinctly different. The measured peak widths of the $\left(\frac{1}{3} \frac{1}{3}\right)$ peaks are 2.07 and $2.87 \%$ of the Brillouin zone for the low and high temperature $\sqrt{3}$ alloy, respectively. Compared to previously published data for the peak widths [29], this indicates that the absolute coverage of the $\sqrt{3}$ alloy phase at the 


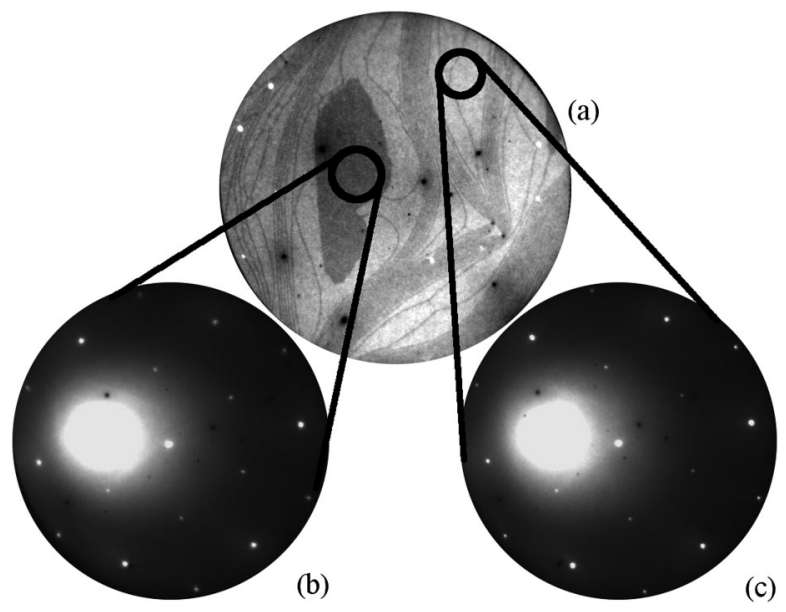

FIG. 2. (a) A $50 \mu \mathrm{m}$ FoV bright-field LEEM image acquired at an energy of $6.3 \mathrm{eV}$ showing the coexistence of the low temperature (bright) and high temperature (dark) $\sqrt{3}$ alloy phases at $537 \mathrm{~K}$. (b) and (c) The $\mu$ LEED patterns of both phases. The patterns were recorded at an electron energy of $49 \mathrm{eV}$ and both reveal a $\sqrt{3} \times \sqrt{3}$ structure.

phase transition temperature is indeed close to, but slightly above the coverage of $0.276 \mathrm{ML}$ that is expected for the hard-hexagon order-disorder transition. We note that an exact calibration of the Bi coverage using just the $\sqrt{3}$ phase is difficult to perform since the precise coverage of $\mathrm{Bi}$ in the $\sqrt{3}$ phase at room temperature, the calibration temperature, is not known from LEED or surface $\mathrm{x}$-ray diffraction (SXRD) measurements. Therefore, to obtain a more precise calibration of the deposition rate we made use of the appearance of several other Bi phases that form and that were accurately characterized in SXRD experiments [32,33].

The changing area fractions indicate a variation in the Bi coverage of the $\sqrt{3}$ phase, but do not reveal any details on the atomic positions of the $\mathrm{Bi}$ atoms. To investigate the structural changes in the $\sqrt{3}$ alloy below and above the phase transition, bright-field LEEM intensities were recorded from LEEM images acquired during deposition of $\mathrm{Bi}$ at various temperatures between room temperature and $687 \mathrm{~K}$. The data are shown in Fig. 3(a). The data appear fully inconsistent with any thermally activated process that would reduce the Bi density in the $\sqrt{3}$ phase, which would yield a monotonic change in reflectivity with temperature. The correlation between the different data sets becomes evident however when we realize that the $\mathrm{Cu}(111)$ surface is contracted inwards in the temperature range that we have investigated. It has a tendency to reduce this inward relaxation with increasing temperature [38]. The need to have $\mathrm{Bi}$ incorporated in the first layer diminishes as temperature is increased. This reduced alloying affinity of the $\mathrm{Cu}(111)$ surface can be compensated for in one of two ways. Either by reducing the Bi density in the surface layer [39], or by a further outward relaxation of the embedded $\mathrm{Bi}$ atoms. The former has already been demonstrated to take
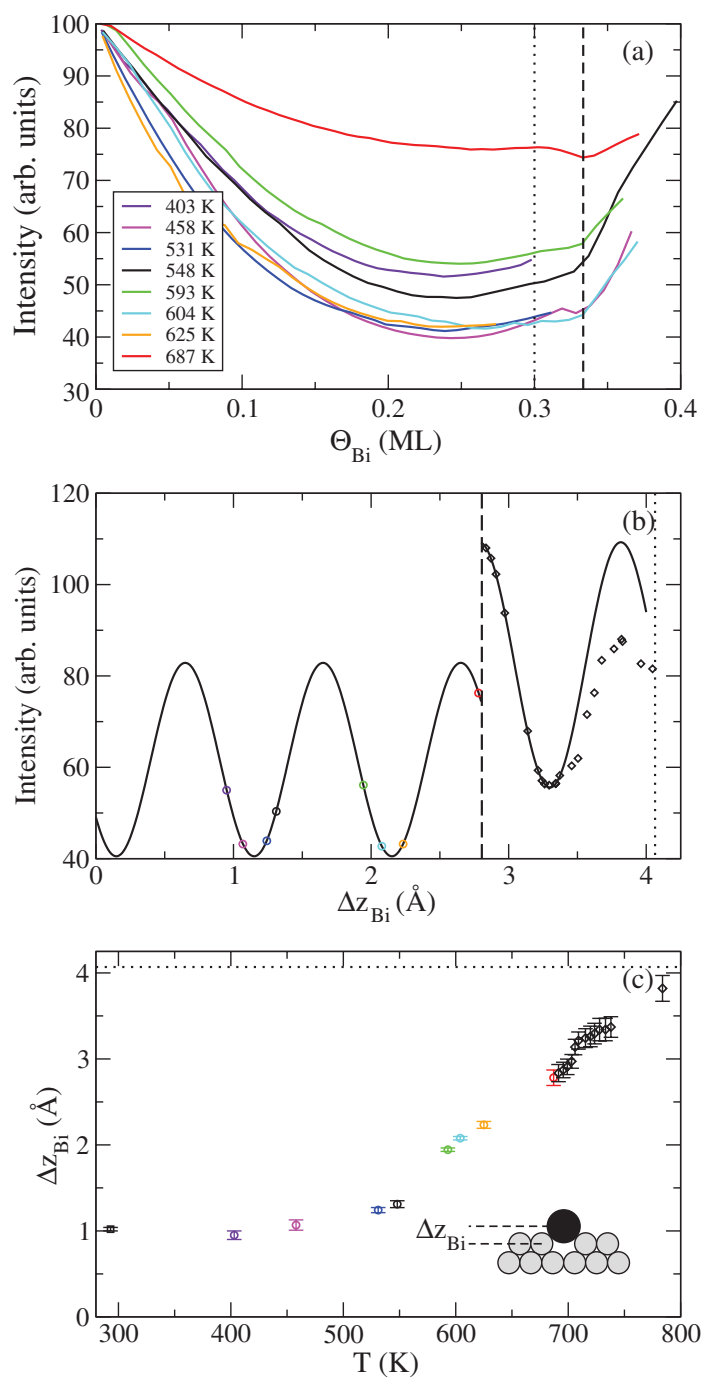

FIG. 3 (color). (a) Bright field intensity of the $\sqrt{3}$ alloy measured as a function of $\mathrm{Bi}$ coverage and temperature at an electron energy of $24 \mathrm{eV}$. (b) Calculated specular reflectivity of the $\sqrt{3}$ alloy phase (solid line) for an electron energy of $24 \mathrm{eV}$ (left of dashed line) and $22 \mathrm{eV}$ (right of dashed line) as a function of $\Delta \mathrm{z}_{\mathrm{Bi}}$. The colored data points are derived from the bright-field intensity data for a $\mathrm{Bi}$ coverage of $0.30 \mathrm{ML}$ shown in (a), others (diamonds) are from a data set recorded at $22 \mathrm{eV}$. The calculated reflected intensity at $22 \mathrm{eV}$ is slightly shifted and has a somewhat smaller period compared to the $24 \mathrm{eV}$ curve. The dotted line indicates the relaxation at which complete dealloying occurs at 805 K (see Fig. 4). (c) Relaxation of embedded Bi as a function of temperature. The data point at room temperature (square) was obtained from SXRD data [32], others from data shown in (b). With the exception of one data point recorded at a maximum in intensity at $3.82 \AA$, z relaxations greater than $3.37 \AA$ could not be accurately measured because of the reduced oscillation amplitude of the bright field intensity at temperatures close to $800 \mathrm{~K}$.

place at a slow rate through Fig. 1(g), the latter can be directly derived from the intensity curves of Fig. 3(a).

Using the precise atomic positions that were derived from previous SXRD measurements [32] the scattering 
factors and corresponding specular reflectivity of the $\sqrt{3}$ alloy phase were calculated for a coverage of $0.30 \mathrm{ML}$ and for increasing values of the Bi relaxation, $\Delta \mathrm{z}_{\mathrm{Bi}}$ (see Supplemental Material Ref. [40]). The vertical position of the first layer $\mathrm{Cu}$ atoms was held constant at the room temperature value. It is unlikely the $\mathrm{Cu}$ atoms will be displaced beyond the bulk position in the temperature range that was investigated [38]. Figure 3(b) shows the calculated bright-field intensities, with the measured intensities from two separate data sets superimposed. The calculated intensities show a clear oscillation with increasing displacement of the $\mathrm{Bi}$ atoms. This is a simple interference effect as the $\mathrm{Bi}$ atoms are displaced further away from the surface. The calculated data is surprisingly insensitive to the position of the $\mathrm{Cu}$ atoms, justifying the choice to leave the position of the $\mathrm{Cu}$ atoms unchanged. The oscillation that is visible in the calculated data is closely followed by the experimental data, but with a reduced amplitude for very large relaxations. The $\mathrm{Bi}$ position that is derived from the data is plotted in Fig. 3(c) versus temperature. Combined with the coverage data, this fully characterizes the structure of the $\sqrt{3}$ phase. Both the atomic positions and composition are known.

The loss of $\mathrm{Bi}$ from the $\sqrt{3}$ phase continues after the order-disorder transition and proceeds concurrently with the outward relaxation of $\mathrm{Bi}$. The decreasing $\mathrm{Bi}$ coverage of the $\sqrt{3}$ alloy is evident from LEEM images shown in Fig. 4. A strong decrease in the area fraction covered by the $\sqrt{3}$ alloy is observed when approaching a temperature of $805 \mathrm{~K}$. Above this temperature the substrate is fully covered by a dilute overlayer phase. The complete expulsion of $\mathrm{Bi}$ from the $\sqrt{3}$ alloy at $805 \mathrm{~K}$ coincides with

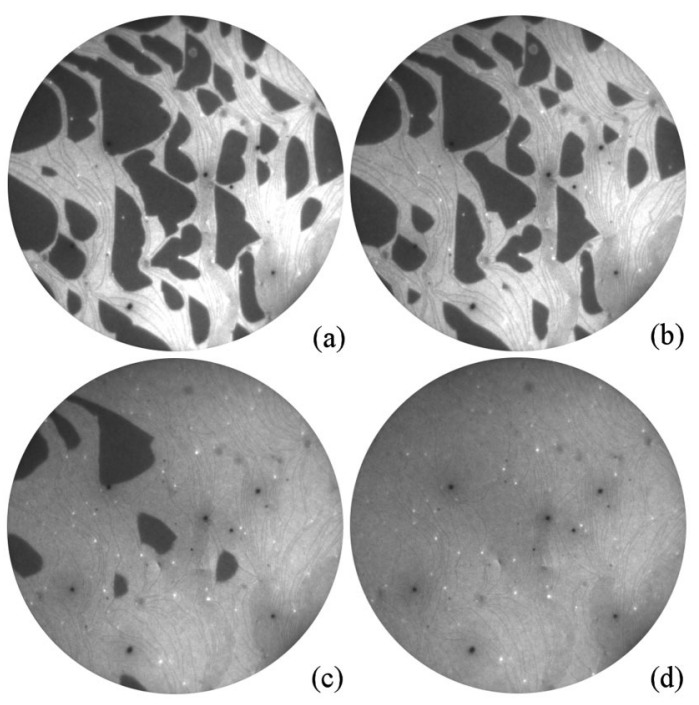

FIG. 4. (a)-(d) $10 \mu \mathrm{m}$ FoV LEEM images recorded at 796, 799 , 802, and $803 \mathrm{~K}$ illustrating the changing relative area fractions of the alloy (dark) and overlayer (bright) phases as the $\sqrt{3}$ phase completely dealloys. the temperature where the $\mathrm{Cu}(111)$ surface interlayer spacing was observed to become equal to the bulk interlayer spacing [38].

Having detailed the temperature dependent structure and dealloying of the $\sqrt{3}$ phase, we propose to interpret our experiments as follows. In the hard-hexagon model, at coverages significantly exceeding the critical coverage $\theta_{c}$ of $0.276 \mathrm{ML}$, effectively only one of three possible sublattices is allowed to be occupied. When the $\sqrt{3}$ phase is forced to coexist with the overlayer phase, a substantial stress mismatch leads to the formation of the domain patterns observed in Figs. 1(a)-1(c) [1,2,5]. As temperature is increased, the $\mathrm{Bi}$ relaxes outwards. The $\mathrm{Bi}$ coverage in the $\sqrt{3}$ alloy also decreases and approaches $\theta_{c}$. The other two sublattices now have a substantial nonzero occupation [41]. This enables the formation of defects in the $\sqrt{3}$ structure, which in turn alters the magnitude of the stress tensor. Just before reaching a Bi coverage of $0.276 \mathrm{ML}$, the rapid change in elastic properties leads to the formation of an apparent miscibility gap of 0.0028 ML. Two $\sqrt{3}$ phases with minutely different coverages, but substantially different elastic properties, coexist, in a manner similar to what occurs in spinodal decomposition [42]. Critical scattering around the critical coverage of $0.276 \mathrm{ML}$ ensures that a $\sqrt{3}$ LEED pattern remains visible [43]. Proceeding to still higher temperatures, the dealloying continues. The $\sqrt{3}$ alloy eventually loses its $\sqrt{3}$ structure completely, as was already hinted at by the increasing FWHMs of the $\sqrt{3}$ diffraction spots in Fig. 2. All three sublattices of the $\sqrt{3}$ phase are now approximately equally occupied. The feature size of the domain patterns is substantially increased as a result of a reduced magnitude of the stress mismatch and the effects of anisotropy on domain shape also become explicitly visible. We propose that dealloying is also responsible for the reduced amplitude of the experimental bright-field intensity oscillations in Fig. 3(b), eventually leading to a complete expulsion of $\mathrm{Bi}$ from the first layer at a temperature just above $800 \mathrm{~K}$, highlighted in Fig. 4.

In summary, we have observed the dealloying of the $\mathrm{Cu}(111)-\sqrt{3} \times \sqrt{3}-R 30^{\circ}-\mathrm{Bi}$ alloy phase. The dealloying occurs through a gradual expulsion of Bi from the $\sqrt{3}$ alloy and goes hand in hand with an outward relaxation of the embedded $\mathrm{Bi}$ atoms. The change in position of the $\mathrm{Bi}$ atoms is directly visible in the electron reflectivity of the $\sqrt{3}$ phase. With increasing temperature, the hard-hexagon critical coverage of $0.276 \mathrm{ML}$ is reached, inducing an order-disorder phase transition in the $\sqrt{3}$ phase. This phase transition enables new mechanisms for elastic relaxation and yields a phase coexistence around a miscibility gap for the $\sqrt{3}$ alloy just above the hard-hexagon critical coverage of 0.276 ML. This gap alters the energetics that govern domain boundary formation in a discontinuous way. In general, any phase transition that enables new pathways for strain relief in a surface phase may discontinuously 
alter elastically stabilized domain patterns involving that phase and thus provides a mechanism to influence the shape and size of self-assembled nanostructures.

We acknowledge Norm Bartelt for many useful and stimulating discussions.

[1] O. L. Alerhand, D. Vanderbilt, R. D. Meade, and J.D. Joannopoulos, Phys. Rev. Lett. 61, 1973 (1988).

[2] D. Vanderbilt, Surf. Sci. Lett. 268, L300 (1992).

[3] V. Zielasek, F. Liu, Y. Zhao, J. B. Maxson, and M. G. Lagally, Phys. Rev. B 64, 201320(R) (2001).

[4] R. Plass, J. A. Last, N. C. Bartelt, and G. L. Kellogg, Nature (London) 412, 875 (2001).

[5] J. B. Hannon, J. Tersoff, and R. M. Tromp, Science 295, 299 (2002).

[6] R. van Gastel, R. Plass, N. C. Bartelt, and G. L. Kellogg, Phys. Rev. Lett. 91, 055503 (2003).

[7] H. J. W. Zandvliet, R. van Gastel, O. Gürlü, and B. Poelsema, Phys. Lett. A 326, 457 (2004).

[8] N. V. Medhekar, V. B. Shenoy, J. B. Hannon, and R. M. Tromp, Phys. Rev. Lett. 99, 156102 (2007).

[9] N. V. Medhekar and V. B. Shenoy, J. Appl. Phys. 103, 063523 (2008).

[10] N. V. Medhekar, V.B. Shenoy, J.B. Hannon, and R. M. Tromp, Appl. Phys. Lett. 91, 253101 (2007).

[11] J. Tersoff and R. M. Tromp, Phys. Rev. Lett. 70, 2782 (1993).

[12] A. Li, F. Liu, and M. G. Lagally, Phys. Rev. Lett. 85, 1922 (2000).

[13] G. E. Thayer, J. B. Hannon, and R. M. Tromp, Nature Mater. 3, 95 (2004).

[14] R. van Gastel, N. C. Bartelt, and G. L. Kellogg, Phys. Rev. Lett. 96, 036106 (2006).

[15] H. J. W. Zandvliet and R. van Gastel, Phys. Rev. Lett. 99, 136103 (2007).

[16] R. van Gastel, N. C. Bartelt, P. J. Feibelman, F. Léonard, and G. L. Kellogg, Phys. Rev. B 70, 245413 (2004).

[17] J. de la Figuera, F. Léonard, N. C. Bartelt, R. Stumpf, and K. F. McCarty, Phys. Rev. Lett. 100, 186102 (2008).

[18] V. I. Marchenko, JETP Lett. 33, 381 (1981).

[19] L.D. Landau and E. M. Lifshitz, Course of Theoretical Physics, Theory of Elasticity Vol. 7 (Pergamon, New York, 1959).
[20] K.-O. Ng and D. Vanderbilt, Phys. Rev. B 52, 2177 (1995).

[21] K. Kern, H. Niehus, A. Schatz, P. Zeppenfeld, J. Goerge, and G. Comsa, Phys. Rev. Lett. 67, 855 (1991).

[22] P. Zeppenfeld, M.A. Krzyzowski, Ch. Romainczyk, R. David, G. Comsa, H. Röder, K. Bromann, H. Brune, and K. Kern, Surf. Sci. 342, L1 131 (1995).

[23] K. Pohl, M.C. Bartelt, J. de la Figuera, N. C. Bartelt, J. Hrbek, and R. Q. Hwang, Nature (London) 397, 238 (1999).

[24] H. Ellmer, V. Repain, S. Rousset, B. Croset, M. Sotto, and P. Zeppenfeld, Surf. Sci. 476, 95 (2001).

[25] G. E. Thayer, V. Ozolins, A. K. Schmid, N. C. Bartelt, M. Asta, J. J. Hoyt, S. Chiang, and R. Q. Hwang, Phys. Rev. Lett. 86, 660 (2001).

[26] F.-J. Meyer zu Heringdorf, Th. Schmidt, S. Heun, R. Hild, P. Zahl, B. Ressel, E. Bauer, and M. Horn-von Hoegen, Phys. Rev. Lett. 86, 5088 (2001).

[27] J. B. Hannon, F.-J. Meyer zu Heringdorf, J. Tersoff, and R. M. Tromp, Phys. Rev. Lett. 86, 4871 (2001).

[28] R. Plass, N. C. Bartelt, and G. L. Kellogg, J. Phys. Condens. Matter 14, 4227 (2002).

[29] R. van Gastel, D. Kaminski, E. Vlieg, and B. Poelsema, Surf. Sci. 603, 3292 (2009).

[30] F. Delamare and G. E. Rhead, Surf. Sci. 35, 172 (1973).

[31] F. Delamare and G. E. Rhead, Surf. Sci. 35, 185 (1973).

[32] D. Kaminski, P. Poodt, E. Aret, N. Radenovic, and E. Vlieg, Surf. Sci. 575, 233 (2005).

[33] D. Kaminski, P. Poodt, E. Aret, N. Radenovic, and E. Vlieg, Phys. Rev. Lett. 96, 056102 (2006).

[34] E. Bauer, Rep. Prog. Phys. 57, 895 (1994).

[35] R. J. Baxter, J. Phys. A 13, L61 (1980).

[36] R. J. Baxter and S. K. Tsang, J. Phys. A 13, 1023 (1980).

[37] R. J. Baxter and P. A. Pearce, J. Phys. A 15, 897 (1982).

[38] K. H. Chae, H. C. Lu, and T. Gustafsson, Phys. Rev. B 54, 14082 (1996).

[39] K. L. Man, Y. J. Feng, C. T. Chan, and M. S. Altman, Surf. Sci. 601, L95 (2007).

[40] See Supplemental Material at http://link.aps.org/ supplemental/10.1103/PhysRevLett.109.195501 for details of the reflectivity calculations.

[41] G. S. Joyce, J. Phys. A 21, L983 (1988).

[42] J. W. Cahn, Acta Metall. 10, 179 (1962).

[43] M. F. Reedijk, J. Arsic, D. Kaminski, P. Poodt, H. Knops, P. Serrano, G. R. Castro, and E. Vlieg, Phys. Rev. B 67, 165423 (2003). 